\title{
European Standards of Care for Newborn Health
}

\section{Natascia Bertoncelli}

Developmental Therapist, NIDCAP Trainer, Neonatal Intensive Care Unit, University Hospital of Modena, Modena, Italy

Urvival rate for preterm infants is continuously improving $\checkmark$ thanks to advances in medical science. Despite this, preterm birth is still a challenge worldwide. The treatment for preterm and ill newborn infants is very complex and it requires specially trained healthcare professionals. In Europe, there is variation in the provision of care for preterm and ill newborn infants at a national, regional, and hospital level including the education available for healthcare professionals. Moreover, national guidelines, when they do exist, vary from country to country.

The European Standards of Care for Newborn Health (ES$\mathrm{CNH}$ ) is an interdisciplinary collaboration project that addressed the disparities in provision and quality of care through the development, and now publication of standards of care for key topics associated with preterm birth and neonatal morbidity. Eleven areas were selected to address, the so-called Topic Expert Groups or TEG (see Figure 1), in which the standards were developed and looked beyond (medical) care of infants and included NICU design, follow-up and continuing care, infant- and family-centred developmental care, and ethical-decision making. ESCNH is a true patient-centred project, and for the first time, patients were involved in every step in the development of standards. In collaboration with parent representatives from more than 30 countries, there were about 220 healthcare professionals from different practice areas involved and worked over several years to create the standards. The standards were launched at the European Parliament in Brussels in November 2018. By that time, 108 healthcare societies and associations as well as 50 parent organisations accepted the European Foundation for the Care of Newborn Infants (EFCNI) invitation and officially supported the newly developed standards. The ESCNH help support the rights of the child of the UN Convention Assembly by serving as a reference for the development and implementation of standards and guidelines on a national and international level.

I personally took part on the standard in Infant and FamilyCentred Developmental Care (IFCDC). The role of the Topic Expert Group on IFCDC was defining practice standards for the implementation of newborn care centred around the infant and his/her family in order to support optimal health and development of preterm and ill newborn infants. This standard is divided in another 10 sub-topics and I was involved mainly in the "Education and training for infant- and family-centred developmental care" section. My group was comprised of a multidisciplinary team from different countries in Europe and we worked together, exchanging hundreds of emails over several years. Our aim was developing educational pathways that ensure that all NICU professionals have educational and training opportunities to develop the knowledge and skills needed to implement high quality infant and family-centred developmental care, which includes guiding of parents as primary caregivers. It was a huge task and incredibly satisfiying. I had the chance to work with amazing people that included sharing ideas and reflections. Each email shared was an opportunity to recalibrate my attention on preterm infants and their parents who I care for in my NICU, and I learned a lot.

All the NIDCAP professionals in my team in Modena, and myself, are working to spread the European Standards of Care for Newborn Health in all the NICUS in Italy. This project and the work that came out of the developed European Standards of Care for Newborn Health is a powerful tool to provide all preterm infants and their parents with the best possible care.

FIGURE 1. The 11 Topic Expert Groups

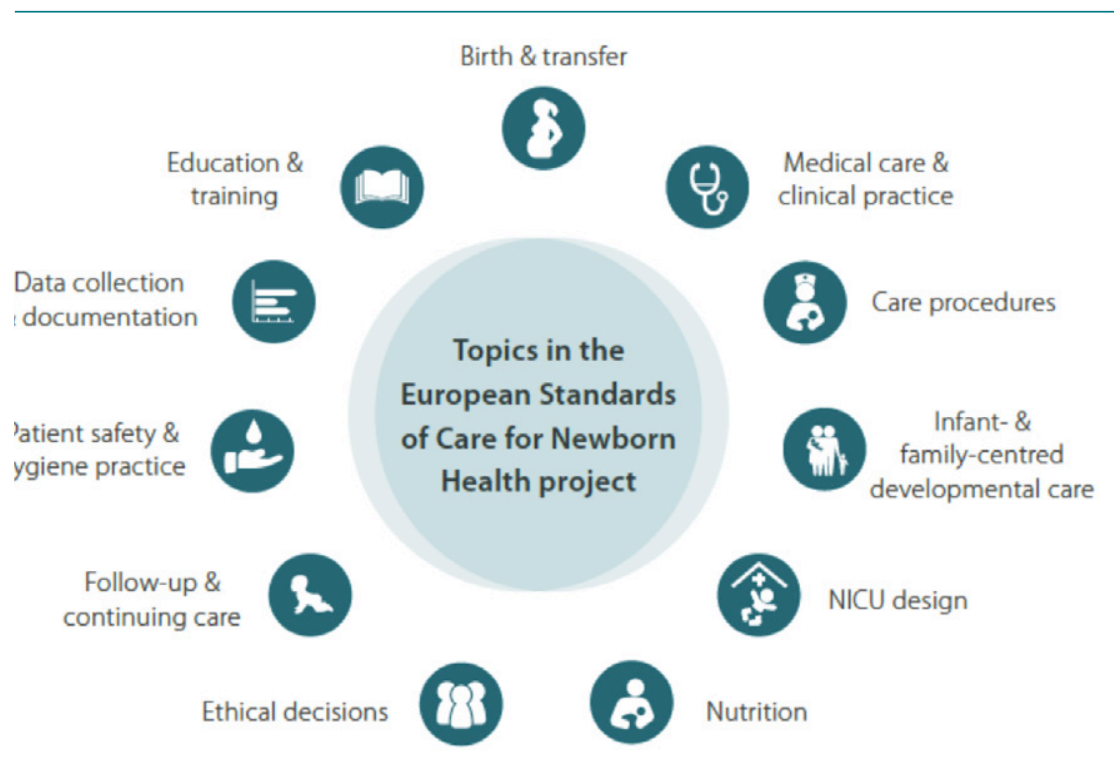

$20 \cdot 2020 \cdot$ Developmental Observer 